\section{Farmacogenómica de los anticoagulantes orales: la importancia de establecer algoritmos de dosificación en población chilena}

\author{
NEFTALÍ GUZMÁN ${ }^{1, \mathrm{a}}$, MARIO VEGA ${ }^{1, \mathrm{~b}}$, FRANCO REYES $^{1, \mathrm{~b}}$, \\ DINA GUZMÁN-OYARZO ${ }^{2, \mathrm{c}}$, MARCELA ANDAUR ${ }^{1, \mathrm{~d}}$, \\ RODRIGO BOGUEN ${ }^{1, a}$, PABLO LETELIER ${ }^{1, a}$
}

\section{Pharmacogenomics of vitamin $\mathrm{K}$ antagonists}

There is an important interindividual variability in dose requirement for coumarinic anticoagulants, which could be explained by genetic and non-genetic factors. Among hereditary factors, there are gene polymorphisms that code the therapeutic target and the main enzyme responsible for their metabolism. However, there are other candidate genes that could modulate dose requirements. The is a paucity of pharmacogenomic platforms to determine dose requirements of coumarinics in the Chilean population. Therefore, algorithms considering different variables to adjust individual dosages are required. Herein, we analyze the available evidence about factors that can modify the effects of vitamin K antagonists and that should be incorporated to dosing algorithms.

(Rev Med Chile 2020; 148: 1307-1314)

Key words: Anticoagulants; Coumarins; Pharmacogenomic Variants.
'Laboratorio de Investigación en Salud de Precisión, Departamento de Procesos Diagnósticos y Evaluación, Facultad de Ciencias de la Salud, Universidad Católica de Temuco. Temuco, Chile.

${ }^{2}$ Centro de Biología Molecular y Farmacogenética, Núcleo de Desarrollo Científico-Tecnológico en Biorecursos, Universidad de La Frontera (BIORENUFRO), Chile.

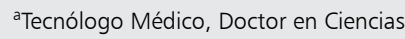
(PhD).

'Tecnólogo Médico, Licenciado en Tecnología Médica.

'Tecnólogo Médico, Doctor ${ }^{\circledR}$ en Ciencias (PhD).

'Tecnólogo Médico, Magíster en Ciencias.

Este trabajo fue apoyado por proyecto FEQUIP2018- PL-06, de la Vicerrectoría de Investigación y Postgrado de la Universidad Católica de Temuco. La institución no tuvo influencia en la preparación, revisión o aprobación del manuscrito.

Los autores declaran no tener conflictos de interés.

Recibido el 29 de julio de 2019, aceptado el 18 de marzo de 2020.

Correspondencia a:

Dr. Pablo Letelier

Manuel Montt 56. Temuco, Chile.

pletelier@uct.cl

\section{I}

os cumarínicos o antagonistas de la vitamina $\mathrm{K}$ son fármacos ampliamente prescritos en el mundo ${ }^{1}$, utilizados para el tratamiento y prevención de eventos tromboembólicos, tanto arteriales como venosos, siendo acenocumarol el fármaco utilizado en diversos países de América Latina. Si bien su dosificación inicial se establece a partir de la International Normalized Ratio (INR), un indicador ampliamente utilizado para evaluar la calidad de la terapia anticoagulante corresponde al tiempo en rango terapéutico (TTR), siendo considerado óptimo por The National Institute for Health and Care Excellence un TTR $>65 \%{ }^{2}$. En Chile, un reciente estudio que recoge información respecto de la eficacia de la terapia anticoagulante muestra que el TTR observado en una cohorte de pacientes fue de $50 \%$, valor por debajo de lo recomendado internacionalmente.

Los cumarínicos actúan a nivel hepático inhibiendo la enzima microsomal vitamina K epóxido reductasa complejo 1 (VKORC1) participando en la reducción de la forma epóxido de la vitamina $\mathrm{K}(\mathrm{KO})$ hacia la forma reducida hidroquinona $\left(\mathrm{KH}_{2}\right)$ e inhibiendo el ciclo de interconversión de la vitamina $\mathrm{K}^{3}$. La forma reducida de la vitamina K participa como cofactor para la $\gamma$-carboxilación de las proteínas de la hemostasia dependientes de vitamina $\mathrm{K}$ (Figura 1) y la ausencia de esta modificación postraduccional genera productos no funcionales que son incapaces de unir calcio, dando como resultado una disminución en la actividad de estos factores de la coagulación ${ }^{3}$.

Evidencia científica da cuenta de una importante variabilidad interindividual en los reque- 
rimientos de dosis de cumarínicos, fenómeno que puede ser explicado por factores genéticos y no genéticos, destacando entre estos factores a variaciones de secuencia en genes candidatos, edad, género, peso corporal, interacción con otros medicamentos, ingesta de vitamina $\mathrm{K}$ en la dieta, tabaquismo ${ }^{1,4}$. El impacto de estas variables en el período de establecimiento de dosis es particularmente importante, por lo que el uso de algoritmos de predicción de dosis de cumarínicos basados en factores genéticos y no genéticos contribuiría a mejorar la seguridad del paciente.

\section{Factores genéticos}

Se ha propuesto un importante número de genes candidatos que explicarían las diferencias en los requerimientos de dosis, sin embargo, gran parte de esta variabilidad estaría asociada a polimorfismos en los genes que codifican, por una parte para la proteína que constituye el blanco terapéutico de los cumarínicos denominada vitamina $\mathrm{K}$ epóxido reductasa (VKORC1), como así también para la enzima que participa en el metabolismo del fármaco denominada citocromo P450 isoforma 2C9 (CYP2C9) ${ }^{5,6}$.

La enzima VKORC1 corresponde al blanco terapéutico de los cumarínicos y se encuentra ubicada en el lumen del retículo endoplásmico de hepatocitos. El gen que codifica para esta enzima, denominado VKORC1, se encuentra en el brazo corto del cromosoma 16 (16p11.2) ${ }^{7}$. La evidencia disponible sugiere que cerca de $40 \%$ de la variabilidad en los requerimientos de dosis de cumarínicos podría ser explicada por cambios de una sola base nitrogenada en este gen ${ }^{6-8}$. Estas variaciones de secuencia que se denominan polimorfismos de nucleótido simple (SNPs), se encuentran relacionadas en algunos casos con sensibilidad y en otros con resistencia al efecto del fármaco. En el grupo de variantes asociadas a sensibilidad al fármaco, el principal SNP descrito corresponde a una sustitución de nucleótidos de guanina por adenina en la posición -1639 de la región promotora del gen (VKORC1 -1639 G>A; rs9923231). Estudios realizados en diferentes grupos poblacionales demuestran significativamente que sujetos portadores del alelo A de este SNP requieren de menores dosis de warfarina y acenocumarol para lograr su efecto antitrombó- tico ${ }^{9,10}$, en comparación con aquellos que poseen el alelo G. Similar asociación se ha descrito para la variante VKORC1 $1173 \mathrm{C}>\mathrm{T}$ (rs9934438), ubicada en la región intrónica del gen ${ }^{11}$. En contraste, la variante alélica VKORC1 3730 G>A (rs7294), ubicada en la región 3'-UTR ha demostrado que aquellos pacientes que presentan al menos un alelo A requieren mayores dosis de cumarínicos que aquellos portadores del alelo $\mathrm{G}^{12}$.

Por su parte, la enzima citocromo $\mathrm{P} 450$ isoforma 2C9 (CYP2C9) es la principal encargada del metabolismo de los cumarínicos en el hígado, siendo CYP2C9 el gen que la codifica, el cual se localiza en el brazo largo del cromosoma 10 (10q24.2) ${ }^{13}$. Polimorfismos en CYP2C9 constituyen uno de los mayores determinantes de la variabilidad en la respuesta a la terapia con cumarínicos, destacando los alelos $C Y P 2 C 9^{\star} 2$ y $C Y P 2 C 9^{*} 3^{1,14}$. La variante CYP2C9² (430C $>$ T; rs1799853) corresponde a una mutación puntual en el exón 3 , siendo responsable del cambio aminoacídico de arginina por cisteína en la posición 144 (Arg144Cys) de CYP2C9, lo que genera una disminución de 30\% de la actividad catalítica de la enzima ${ }^{15}$. Por su parte, la variante $C Y P 2 C 9^{*} 3(1075 \mathrm{~A}>C$; rs 1057910$)$, es resultado de una mutación en el exón 7 del gen, causando una sustitución en la posición Ile359Leu y produciendo una disminución de $80 \%$ de la actividad enzimática de CYP2C9, lo que se ha asociado a un incremento del riesgo hemorrágico en individuos portadores, debido a que existiría mayor disponibilidad de cumarínicos para inhibir a VKORC1 $1^{15,16}$.

Si bien la variabilidad hereditaria observada en la terapia anticoagulante oral puede ser explicada principalmente por polimorfismos en los genes VKORC1 y CYP2C9, no obstante, esta variabilidad en la respuesta se ha asociado en menor medida a otros genes candidatos como $\gamma$-glutamil carboxilasa ( $G G C x$ ), citocromo P450 isoforma $4 \mathrm{~F} 2(C Y P 4 F 2)$ y calumenina $(C A L U)^{17,18}$. El gen $G G C x$ codifica para la enzima gama-glutamil carboxilasa (GGCx) que corresponde a una proteína encargada de catalizar la $\gamma$-carboxilación de los factores vitamina $\mathrm{K}$ dependientes. Algunos estudios demuestran que SNPs en GGCx estarían asociados a cambios en los requerimientos de dosis de cumarínicos ${ }^{19,20}$. Por su parte, el gen CYP4F2 codifica para la enzima citocromo P450 isoforma $4 \mathrm{~F} 2$, que corresponde a una vitamina $\mathrm{K}_{1}$ oxidasa, que puede inactivar a la vitamina $\mathrm{K}_{1}$ 
por hidroxilación, disminuyendo la vitamina $\mathrm{K}$ hidroquinona para la $\gamma$-carboxilación de los factores de coagulación dependientes de vitamina $\mathrm{K}$. La principal variante descrita corresponde a CYP4F2 1347G $>$ A (rs2108622) que ha sido asociada a mayores requerimientos de dosis para alcanzar el objetivo terapéutico del anticoagulante ${ }^{18,21,22}$. Finalmente, el gen $C A L U$ codifica para calumenina, que corresponde a una proteína chaperona que se encuentra en el retículo endoplasmático, la cual actúa generando una regulación de la biosíntesis de proteínas dependientes de vitamina $\mathrm{K}$ de acuerdo a estudios en modelo animal ${ }^{23}$. En cuanto a este gen, se ha demostrado que sujetos portadores de la variante $C A L U$ rs339097 presentan requerimientos significativamente mayores de warfarina respecto a sujetos que no poseen el alelo mutado ${ }^{24}$.
El mecanismo de acción de los cumarínicos y algunos genes candidatos asociados a variabilidad en los requerimientos de dosis de antagonistas de la vitamina K se presentan en la Figura 1.

\section{Factores no genéticos}

Diversos factores han sido propuestos para explicar, en parte, la variabilidad no hereditaria observada en los requerimientos de dosis de antagonistas de la vitamina $\mathrm{K}$, entre los que destacan la edad, género, dieta y etnicidad.

Se ha establecido que la edad constituiría un factor determinante en la variabilidad de los requerimientos de dosis de cumarínicos, lo que explicaría aproximadamente $14,6 \%$ de la variabili-

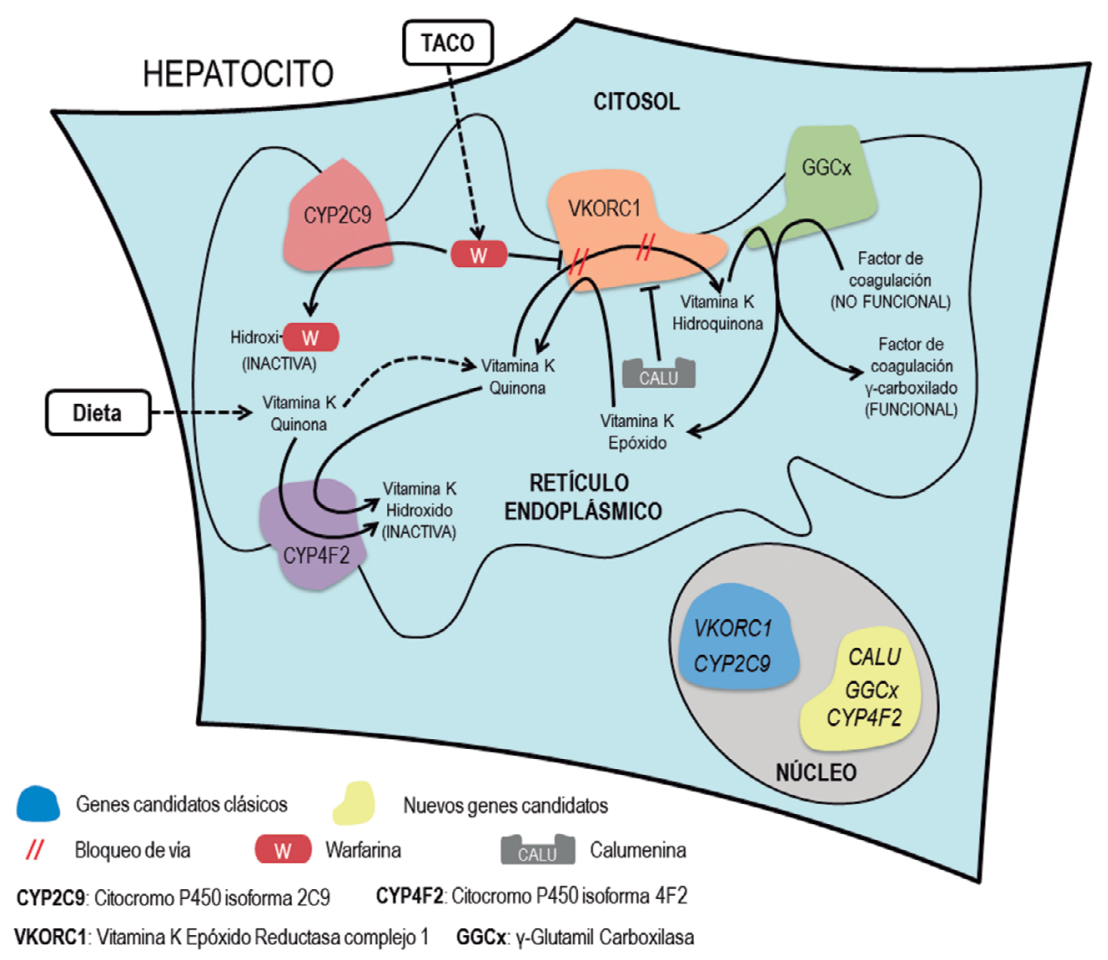

Figura 1. Mecanismo de acción de cumarínicos y genes candidatos asociados a variabilidad en los requerimientos de dosis de antagonistas de la vitamina K. La vitamina K de la dieta es metabolizada por VKORC1 en el retículo endoplásmico del hepatocito formando vitamina K hidroquinona, que actúa como cofactor para GGCx, enzima que cataboliza la $\gamma$-carboxilación de regiones altamente conservadas de proteínas de la hemostasia dependientes de vitamina $K$, permitiendo que sean funcionales. Durante la terapia anticoagulante oral (TACO), los cumarínicos como la warfarina inactivan a VKORC1, impidiendo el ciclo de metabolización de la vitamina K. Normalmente, la enzima CYP2C9 metaboliza a warfarina formando hidroxiwarfarina, una forma inactiva del fármaco. Diversos genes han sido propuestos como moduladores de la variabilidad en la respuesta al tratamiento, destacando VKORC1 y CYP2C9. Otros genes candidatos corresponden a CALU, GGCX y CYP4F2. 
dad observada en sujetos tratados con warfarina ${ }^{25}$. Concordante con esto, la evidencia disponible sostiene la idea que los requerimientos de dosis disminuyen aproximadamente $0,4 \mathrm{mg}$ por año de vida, como consecuencia de una mayor sensibilidad a cumarínicos, incrementando el riesgo de eventos adversos de tipo hemorrágico ${ }^{26}$.

Del mismo modo, género y masa corporal son variables que han demostrado ser predictores independientes en los requerimientos de dosis de antagonistas de la vitamina $K$. En sujetos tratados con warfarina se ha demostrado que el género masculino y una mayor masa corporal se relacionarían con mayores requerimientos de dosis de anticoagulante ${ }^{27}$. Por su parte, respecto del impacto de la alimentación sobre la respuesta terapéutica a cumarínicos, se ha demostrado que la respuesta a la terapia se modifica de acuerdo a la ingesta de alimentos ricos en vitamina $\mathrm{K}$. En este contexto, alimentos que presentan un importante contenido de vitamina $K$ incrementarían los requerimientos del fármaco para alcanzar el objetivo terapéutico ${ }^{28}$.

La etnicidad constituye un importante aspecto a considerar en el establecimiento de dosis en la terapia anticoagulante oral. Se ha establecido que la población asiática presentaría menores requerimientos de dosis de cumarínicos que sujetos caucásicos para alcanzar el objetivo terapéutico, mientras que los requerimientos en sujetos negroides serían mayores a otras poblaciones ${ }^{29}$. Estas diferencias podrían explicarse, a lo menos en parte, por las diferencias en la frecuencia de presentación de SNPs en genes candidatos, donde se observan importantes variaciones poblacionales que explicarían las diferencias en los requerimientos de dosis de cumarínicos ${ }^{30}$. En este contexto, las poblaciones latinoamericanas se constituyen de una mezcla de poblaciones nativas americanas o amerindias, europeos y negroides, lo que ha sido demostrado utilizando diversas herramientas moleculares, entre ellas marcadores uniparentales como el ADN mitocondrial (mtDNA) ${ }^{31}$. En un estudio realizado en individuos chilenos en terapia con cumarínicos y utilizando marcadores del genoma mitocondrial, previamente hemos demostrado una elevada frecuencia de haplogrupos amerindios de mtDNA, lo que da cuenta de un predominante componente amerindio en la población chilena ${ }^{32}$. Este componente genético, compartido por poblaciones latinoamericanas, enfatiza la necesidad de evaluar si variantes genéticas descritas en poblaciones caucásicas, negroides o asiáticas, podrían ser considerados biomarcadores de respuesta a tratamiento anticoagulante en nuestra población.

\section{Algoritmos de dosificación para antagonistas de la vitamina $K$}

El desarrollo de algoritmos de dosificación, que consideren factores genéticos y no genéticos, constituye una aplicación que muestra un impacto significativo en la clínica de la terapia anticoagulante oral ${ }^{1,5,33}$. Este nuevo abordaje permite una mayor precisión, especialmente durante el período de establecimiento de dosis, mejorando la respuesta terapéutica y disminuyendo la probabilidad de eventos adversos ${ }^{34}$. A partir de esta evidencia, la Food and Drug Administration de los Estados Unidos de Norteamérica (FDA) sugiere la realización de estudios farmacogenómicos al momento de prescribir cumarínicos.

Respecto de los biomarcadores moleculares mayormente descritos en el mundo asociados a variabilidad en la respuesta terapéutica a cumarínicos, la Tabla 1 muestra la frecuencia de presentación de variantes en genes candidatos observada en estudios realizados en población latinoamericana y su efecto respecto de los requerimientos de dosis del fármaco. En Chile existe limitada evidencia del impacto de SNPs en genes candidatos asociados a la terapia anticoagulante oral. Un estudio realizado en 60 usuarios chilenos tratados con warfarina y 125 individuos en terapia con acenocumarol demostró que la dosis de mantenimiento presentaba una fuerte asociación con la variante VKORC1 -1639G >A (rs9923231) determinando menores requerimientos del fármaco, no encontrándose asociación con variantes en $C Y P 2 C 9^{36}$. Estos resultados son consistentes con otro estudio nacional realizado en individuos tratados con acenocumarol, donde se demuestra una elevada frecuencia de presentación de la variante VKORC1 -1639 G>A (rs9923231), la cual se encuentra asociada a menores requerimientos del fármaco para lograr el efecto terapéutico. En contraste, no se demostró asociación significativa del polimorfismo CYP2C9 $9^{\star}$ (rs1799853) con variabilidad en los requerimientos de dosis del acenocumarol $^{40}$.

Dos importantes estudios han propuesto al- 
Tabla 1. Frecuencia de presentación de variantes en genes candidatos y su asociación con variabilidad en los requerimientos de dosis de cumarínicos en Latinoamérica

\begin{tabular}{|c|c|c|c|c|c|c|}
\hline \multirow{4}{*}{$\begin{array}{l}\text { Variante } \\
\text { CYP2C }{ }^{*} 2 \\
430 \text { C > T } \\
\text { (rs1799853) }\end{array}$} & \multirow{2}{*}{$\begin{array}{l}\text { País } \\
\text { Colombia }\end{array}$} & \multirow{2}{*}{$\begin{array}{c}\mathbf{n} \\
130\end{array}$} & \multicolumn{2}{|c|}{ Frecuencia alélica } & \multirow{2}{*}{$\begin{array}{c}\text { Efecto } \\
\text { No se observa }\end{array}$} & \multirow{2}{*}{$\begin{array}{c}\text { Referencia } \\
\text { [35] }\end{array}$} \\
\hline & & & C & 0,92 & & \\
\hline & & & $\mathrm{T}$ & 0,08 & & \\
\hline & Chile & 170 & C & 0,888 & No se observa & [36] \\
\hline & & & $\mathrm{T}$ & 0,112 & & \\
\hline & Brasil & 198 & C & 0,87 & Menor dosis & {$[37]$} \\
\hline & & & $\mathrm{T}$ & 0,13 & & \\
\hline & Argentina & 101 & C & 0,74 & No reportado & [38] \\
\hline & & & $\mathrm{T}$ & 0,26 & & \\
\hline & Puerto Rico & 163 & C & 0,89 & Menor dosis & [39] \\
\hline & & & $\mathrm{T}$ & 0,11 & & \\
\hline \multirow{10}{*}{$\begin{array}{l}\text { CYP2C9*3 } \\
1075 \mathrm{~A}>C \\
(\text { rs1057910) }\end{array}$} & Colombia & 130 & A & 0,95 & Menor dosis & [35] \\
\hline & & & C & 0,05 & & \\
\hline & Chile & 170 & A & 0,941 & No se observa & {$[36]$} \\
\hline & & & C & 0,059 & & \\
\hline & Brasil & 198 & A & 0,92 & Menor dosis & [37] \\
\hline & & & C & 0,08 & & \\
\hline & Argentina & 101 & A & 0,97 & No reportado & [38] \\
\hline & & & C & 0,03 & & \\
\hline & Puerto Rico & 163 & A & 0,96 & Menor dosis & [39] \\
\hline & & & C & 0,04 & & \\
\hline \multirow{10}{*}{$\begin{array}{l}\text { VKORC1 } \\
-1639 \text { G>A } \\
(\text { rs9923231) }\end{array}$} & Colombia & 130 & G & 0,43 & Menor dosis & [35] \\
\hline & & & A & 0,57 & & \\
\hline & Chile & 170 & G & 0,5 & Menor dosis & {$[36]$} \\
\hline & & & A & 0,5 & & \\
\hline & Brasil & 198 & G & 0,65 & Menor dosis & [37] \\
\hline & & & A & 0,35 & & \\
\hline & Argentina & 101 & G & 0,5 & No reportado & [38] \\
\hline & & & A & 0,5 & & \\
\hline & Puerto Rico & 163 & G & 0,65 & Menor dosis & [39] \\
\hline & & & A & 0,35 & & \\
\hline
\end{tabular}

CYP2C9*2 = Citocromo P450 isoforma 2C9, alelo 2; CYP2C9*3 = Citocromo P450 isoforma 2C9, alelo 3; VKORC1 = vitamina K epóxido reductasa, complejo 1.

goritmos de dosificación para acenocumarol. El primero de ellos logró establecer un algoritmo de dosis guiada por genotipo en una cohorte de 375 pacientes tratados con acenocumarol en Holanda, permitiendo explicar 49\% de la variabilidad en los requerimientos de dosis ${ }^{41}$. Por su parte, un estudio realizado en población española estableció un algoritmo basado en variables clínicas y presencia de polimorfismos en los genes VKORC1, CYP2C9,
CYP4F2 y $A P O E^{10}$. Al analizar estudios farmacogenómicos aplicados a la terapia anticoagulante oral en población latinoamericana, la Tabla 2 presenta algunos algoritmos de dosis establecidos para esta población, que contribuirían a explicar entre $46 \%$ y $70 \%$ de la variabilidad en los requerimientos de dosis de warfarina en las poblaciones evaluadas. Si bien la evidencia en población latinoamericana es escasa, variantes en genes VKORC1 y CYP2C9 
Tabla 2. Algoritmos de dosis guiados por farmacogenómica aplicados a la terapia anticoagulante con warfarina en población latinoamericana

\begin{tabular}{|c|c|c|c|c|c|}
\hline Población & $\mathbf{n}$ & Factores genéticos & Factores no genéticos & $\mathbf{R}^{2}$ & Referencia \\
\hline Puerto Rico & 255 & $\begin{array}{c}\text { CYP2C9*2 } \\
\text { CYP2C9*3 } \\
\text { CYP2C9*8 } \\
\text { VKORC1 }-1639 \mathrm{G}>\mathrm{A} \\
\text { CYP4F2 } 1347 \mathrm{C}>\mathrm{T} \\
\text { NQO1 } 559 \mathrm{C}>\mathrm{T}\end{array}$ & $\begin{array}{c}\text { Edad, INR/dosis, } \\
\text { amiodarona }\end{array}$ & 0,70 & [42] \\
\hline Puerto Rico & 163 & $\begin{array}{c}\text { CYP2C9*2 } \\
\text { CYP2C9*3 } \\
\text { CYP2C9*5 } \\
\text { VKORC1 }-1691 \mathrm{G}>\mathrm{A}\end{array}$ & $\begin{array}{l}\text { Edad, EP, INR/dosis, } \\
\text { amiodarona }\end{array}$ & 0,67 & [39] \\
\hline Brasil & 279 & $\begin{array}{c}\text { CYP2C9*2 } \\
\text { CYP2C9*3 } \\
\text { VKORC1 }-1691 \mathrm{G}>\mathrm{A} \\
\text { VKORC1 } 1173 \mathrm{C}>\mathrm{T} \\
\text { VKORC1 } 3730 \mathrm{G}>\mathrm{A} \\
\text { CYP4F2 } 1347 \mathrm{C}>\mathrm{T} \\
\text { F2 } 494 \mathrm{C}>\mathrm{T}\end{array}$ & $\begin{array}{c}\text { Edad, peso, fármacos } \\
\text { concomitantes (amiodarona, } \\
\text { diureticos, carbamazepina, } \\
\text { bloqueadores } \beta \text {-adrenergicos, } \\
\text { amlodipino }\end{array}$ & 0,63 & [37] \\
\hline Colombia & 152 & $\begin{array}{c}\text { CYP2C9*2 } \\
\text { CYP2C9*3 } \\
\text { VKORC1 }-1691 \mathrm{G}>\mathrm{A} \\
\text { VKORC1 } 1173 \mathrm{C}>\mathrm{T}\end{array}$ & Edad, género & 0,46 & [43] \\
\hline
\end{tabular}

$\mathrm{n}=$ Número de pacientes; $\mathrm{EP}=$ Embolismo pulmonar; INR = Razón normalizada internacional; CYP2C9 = Citocromo P450 isoforma 2C9, CYP4F2 = Citocromo P450 isoforma 4F2; VKORC1 = vitamina K epóxido reductasa, complejo 1; F2 = Factor II de la coagulación (protrombina); NQO1 $=\mathrm{NAD}(\mathrm{P}) \mathrm{H}$ quinona dehidrogenasa 1

explicarían una parte importante de la variabilidad observada en los requerimientos de dosis de cumarínicos. En Chile, un estudio realizado preliminarmente demostró que la variante VKORC1 -1691 G>A, además de factores no genéticos como la edad y peso corporal, fueron asociados de manera independiente a variación en los requerimientos de dosis en pacientes tratados con acenocumarol, lo que permitiría establecer un algoritmo de dosis guiado por farmacogenómica basado en estas variables ${ }^{40}$.

\section{Conclusiones}

La terapia anticoagulante oral requiere un control riguroso en su dosificación para la prevención efectiva de eventos adversos en pacientes, siendo relevante considerar múltiples factores que puedan generar la variabilidad en la respuesta de estos sujetos. Frente a esta problemática, la evidencia científica da cuenta de la utilidad del uso de pla- taformas farmacogenómicas aplicadas a la clínica en la terapia anticoagulante oral, permitiendo mejorar el manejo y pronóstico de los usuarios.

Diversos factores deberían ser considerados para evaluar su potencial aplicación en población chilena. En primer lugar, los modelos de predicción de dosis ya existentes para warfarina pueden no ser aplicables a acenocumarol, principal anticoagulante oral prescrito en Chile, como consecuencia de las diferencias en la farmacocinética entre estos fármacos. Además, los biomarcadores moleculares que muestran un mayor impacto en la clínica presentan importantes variaciones poblacionales, existiendo escasa evidencia de su frecuencia de presentación e impacto en Chile.

Se hace necesario desarrollar estudios en el país que permitan identificar marcadores moleculares y factores no genéticos que pudiesen ser incluidos en algoritmos de dosis para población chilena. Además, la integración de nuevas herramientas como la inteligencia artificial contribuiría aun más a mejorar la capacidad de predicción de los 
algoritmos de dosis. En este sentido, un reciente estudio, que utiliza redes neuronales para construir un algoritmo basado en factores genéticos y clínicos en sujetos tratados con warfarina, logró explicar 93,5\% de la variabilidad en la dosis del fármaco, reduciendo significativamente los INR fuera de rango, la tasa de reacciones adversas a drogas y el tiempo de establecimiento de dosis ${ }^{44}$. Por tanto, la implementación en la clínica de algoritmos de predicción de dosis de anticoagulantes orales, basados en el uso de herramientas de salud de precisión, contribuirían significativamente a mejorar la seguridad de la terapia anticoagulante.

\section{Referencias}

1. Jiménez-Varo E, Canadas-Garre M, Henriques CI, Pinheiro AM, Gutiérrez-Pimentel M, Calleja-Hernández M. Pharmacogenetics role in the safety of acenocoumarol therapy. Thromb Haemost 2014;112 (3): 522-36.

2. Nieto E, Suárez M, Roco A, Rubilar JC, Tamayo F, Rojo $\mathrm{M}$, et al. Anticoagulation Management With Coumarinic Drugs in Chilean Patients. Clin Appl Thromb Hemost 2019; 25: 1-7.

3. Ageno W, Gallus A, Wittkowsky A, Crowther M, Hylek E, Palareti G. Oral anticoagulant therapy: Antithrombotic Therapy and Prevention of Thrombosis. Chest 2012; 141 (2 Suppl): e44S-88S.

4. Zhang J, Wu T, Chen W, Fu J, XXia X, Chen L. Effect of Gene-Based Warfarin Dosing on Anticoagulation Control and Clinical Events in a Real-World Setting. Front Pharmacol 2020; 10: 1527.

5. Tavares LC, Marcatto LR, Santos PCJL. Genotype-guided warfarin therapy: current status. Pharmacogenomics 2018; 19 (7): 667-85.

6. Akram Bader L, Elewa H. The Impact of Genetic and Non-Genetic Factors on Warfarin Dose Prediction in MENA Region: A Systematic Review. PLoS One 2016; 11 (12): e016873.

7. Oldenburg J, Watzka M, Rost S, Muller CR. VKORC1: molecular target of coumarins. J Thromb Haemost 2007; 5 (1): 1-6.

8. Cooper G, Johnson J, Langaee T, Feng H, Stanaway I, Schwarz U, et al. A genome-wide scan for common genetic variants with a large influence on warfarin maintenance dose. Blood 2008; 112 (4): 1022-27.

9. Yuan H, Chen J, Lee M, Wung J, Chen Y, Charng M, et al. A novel functional VKORC1 promoter polymorphism is associated with inter-individual and inter-eth- nic differences in warfarin sensitivity. Hum Mol Genet 2005; 14 (13): 1745-51.

10. Borobia A, Lubomirov R, Ramírez E, Lorenzo A, Campos A, Muñoz-Romo R, et al. An Acenocoumarol dosing algorithm using clinical and pharmacogenetic data in Spanish patients with thromboembolic disease. PLoS One 2012; 7 (7): e41360.

11. Verhoef T, Redekop W, Daly A, van Schie R, de Boer A, Maitland-van der Zee AH. Pharmacogenetic-guided dosing of coumarin anticoagulants: algorithms for warfarin, acenocoumarol and phenprocoumon. Br J Clin Pharmacol 2014; 77 (4): 626-41.

12. Cini $M$, Legnani $C$, Cosmi B, Guazzaloca $G$, Valdrè L, Frascaro $\mathrm{M}$, et al. A new warfarin dosing algorithm including VKORC1 $3730 \mathrm{G}>$ A polymorphism: comparison with results obtained by other published algorithms. Eur J Clin Pharmacol 2012; 68 (8): 1167-74.

13. Magalhães A, Braga K, Afonso E, Pedra R, de Freitas E, Dias D, et al. Algorithm for predicting low maintenance doses of warfarin using age and polymorphisms in genes CYP2C9 and VKORC1 in Brazilian subjects. Pharmacogenomics J 2020; 20 (1): 104-13.

14. Tassies D, Freire C, Pijoan J, Maragall S, Monteagudo J, Ordinas A, et al. Pharmacogenetics of acenocoumarol: cytochrome P450 CYP2C9 polymorphisms influence dose requirements and stability of anticoagulation. Haematologica 2002; 87 (11): 1185-91.

15. Fung E, Patsopoulos N, Belknap S, O’Rourke D, Robb J, Anderson J, et al. Effect of Genetic Variants, Especially CYP2C9 and VKORC1, on the Pharmacology of Warfarin. Semin Thromb Hemost 2012; 38 (8): 893-904.

16. Jorgensen A, FitzGerald R, Oyee J, Pirmohamed M, Williamson P. Influence of CYP2C9 and VKORC1 on Patient Response to Warfarin: A Systematic Review and Meta-Analysis. PLoS One 2012; 7 (8): e44064.

17. Daly AK. Optimal dosing of warfarin and other coumarin anticoagulants: the role of genetic polymorphisms. Arch Toxicol 2013; 87 (3): 407-20.

18. Kaye JB, Schultz LE, Steiner HE, Kittles RA, Cavallari L, Karnes JH. Warfarin Pharmacogenomics in Diverse Populations. Pharmacotherapy 2017; 37 (9): 1150-63.

19. Wadelius M, Chen L, Downes K, Ghori J, Hunt S, Eriksson N, et al. Common VKORC1 and GGCX polymorphisms associated with warfarin dose. Pharmacogenomics J 2005; 5 (4): 262-70.

20. Sun Y, Wu Z, Li S, Qin X, Li T, Xie L, et al. Impact of gamma-glutamyl carboxylase gene polymorphisms on warfarin dose requirement: a systematic review and meta-analysis. Thromb Res 2015; 135 (4): 739-47.

21. Teichert M, Eijgelsheim M, Rivadeneira F, Uitterlinden A, van Schaik R, Hofman A, et al. A genome-wide as- 
sociation study of acenocoumarol maintenance dosage. Hum Mol Genet 2009; 18 (19): 3758-68.

22. Cullell N, Carrera C, Muiño E, Torres N, Krupinski J, Fernández-Cadenas I. Pharmacogenetic studies with oral anticoagulants. Genome-wide association studies in vitamin $\mathrm{K}$ antagonist and direct oral anticoagulants. Oncotarget 2018; 9 (49): 29238-58.

23. Wajih N, Sane D, Hutson S, Wallin R. The inhibitory effect of calumenin on the vitamin K-dependent gamma-carboxylation system. Characterization of the system in normal and warfarin-resistant rats. J Biol Chem 2004; 279 (24): 25276-83.

24. Voora D, Koboldt DC, King CR, Lenzini PA, Eby CS, Porche-Sorbet R, et al. A polymorphism in the VKORC1 regulator calumenin predicts higher warfarin dose requirements in African Americans. Clin Pharmacol Ther 2010; 87 (4): 445-51.

25. Hillman M, Wilke R, Caldwell M, Berg R, Glurich I, Burmester J. Relative impact of covariates in prescribing warfarin according to CYP2C9 genotype. Pharmacogenetics 2004; 14 (8): 539-47.

26. Shendre A, Parmar GM, Dillon C, Beasley TM, Limdi NA. Influence of age on warfarin dose, anticoagulation control, and risk of hemorrhage. Pharmacotherapy 2018; 38 (6): 588-96.

27. Absher RK, Moore ME, Parker MH. Patient-specific factors predictive of warfarin dosage requirements. Ann Pharmacother 2002; 36 (10): 1512-17.

28. Kampouraki E, Kamali F. Dietary implications for patients receiving long-term oral anticoagulation therapy for treatment and prevention of thromboembolic disease. Expert Rev Clin Pharmacol 2017; 10 (8): 789-97.

29. Johnson J. Ethnic Differences in Cardiovascular Drug Response: Potential Contribution of Pharmacogenetics. Circulation 2008; 118: 1383-93.

30. Limdi N, Brown T, Yan Q, Thigpen JL, Shendre A, Liu $\mathrm{N}$, et al. Race influences warfarin dose changes associated with genetic factors. Blood 2015; 126: 539-45.

31. Price A, Patterson N, Yu F, Cox D, Waliszewska A, Gavin J. McDonald GJ, et al. A Genomewide Admixture Map for Latino Populations. Am J Hum Genet 2007; 80: 1024-36.

32. Subiabre V, Palomo I, Guzmán N, Retamales E, Henríquez H, González L. The Influence of Ethnicity on Warfarin Dosage Requirements in the Chilean Population. Curr Ther Res Clin Exp 2015; 77: 31-4.

33. Quiñones L, Roco A, Miranda C. Farmacogenómica: Aplicaciones cardiovasculares. Rev Med Clin Condes 2015; 26 (2): 198-209.
34. Wang L, McLeod H, Weinshilboum RM. Genomics and Drug Response. N Engl J Med 2011; 364: 1144-53.

35. Cifuentes R, Murillo J, Avella E. Predicción de la sensibilidad a la warfarina con base en polimorfismos de los genes VKORC1 y CYP2C9 en pacientes colombianos. Biomédica 2016; 36: 91-100.

36. Benavides F, Grossman N, Poggi H, Nieto E, Bertrán A, Araos D, et al. Efecto de las variantes de VKORC1 y CYP2C9 sobre la dosis de anticoagulantes orales en individuos chilenos. Rev Med Chile 2015; 143 (11): 1369-76.

37. Bottom MR, Bandinelli E, Paim Rohde LE, Amon LC, Hutz MH. Influence of genetic, biological and pharmacological factors on warfarin dose in a Southern Brazilian population of European ancestry. Br J Clin Pharmacol 2011; 72 (3): 442-50.

38. Scibona P, Redal MA, Garfi LG, Arbelbide J, Argibay PF, Belloso WH. Prevalence of CYP2C9 and VKORC1 alleles in the Argentine population and implications for prescribing dosages of anticoagulants. Genet Mol Res 2012; 11 (1): 70-6.

39. Ramos A, Seip RL, Rivera-Miranda G, Felici-Giovanini ME, García-Berdecia R, Alejandro-Cowan Y, et al. Development of a pharmacogenetic-guided warfarin dosing algorithm for Puerto Rican patients. Pharmacogenomics 2012; 13 (16): 1937-50.

40. Guzmán N, Calderón L, Edwards G, Nilson H, Sagrado A, Mella R, et al. Definición de un algoritmo de dosis basado en farmacogenética aplicado a la terapia anticoagulante oral con acenocumarol en población chilena. Rev Urug Cardiol 2010; 25 (2): 160.

41. van Schie R, Wessels J, le Cessie S, de Boer A, Schalekamp T, van der Meer F, et al. Loading and maintenance dose algorithms for phenprocoumon and acenocoumarol using patient characteristics and pharmacogenetic data. Eur Heart J 2011; 32: 1909-17.

42. Duconge J, Ramos AS, Claudio-Campos K, Rivera-Miranda G, Bermúdez-Bosch L, Renta JY, et al. A Novel Admixture-Based Pharmacogenetic Approach to Refine Warfarin Dosing in Caribbean Hispanics. PLoS One 2016; 11 (1): e0145480.

43. Gálvez JM, Restrepo CM, Contreras NC, Alvarado C, Calderón-Ospina CA, Peña N, et al. Creating and validating a warfarin pharmacogenetic dosing algorithm for Colombian patients. Pharmagenom Pers Med 2018; 11 : 169-78.

44. Pavani A, Naushad SM, Kumar RM, Srinath M, Malempati AR, Kutala VK. Artificial neural network-based pharmacogenomic algorithm for warfarin dose optimization. Pharmacogenomics 2016; 17 (2): 121-31. 